\title{
Aprendizaje del inglés a través de tiras cómicas en educación superior ecuatoriana.
}

\begin{abstract}
(요요
\end{abstract}
Learning English through comics strips in Ecuadorian higher education.

Lorena Maribel Yumi Guacho. ${ }^{1}$, José Luis Andrade Mendoza. ${ }^{2}$ \& Yajaira Natali Padilla Padilla. ${ }^{3}$

\begin{abstract}
.
DOI: https://doi.org/10.33262/concienciadigital.v4i1.2.1594

Introduction. Meaningful learning has a great impact on the teaching and learning process in the English language, this study encourages the participation of foreign language students and language teachers to optimize the oral and written production of the English language in the understanding of genuine pedagogical material during the creation of comic strips and English writing. Objective. Demonstrate the motivation of students at the higher level for language development. Encourage the creation of illustrative material to promote foreign language proficiency. Promote oral production through activities such as storytelling and communicative writing. Methodology. The research was conducted through two methods, the first one was based on the qualitative method in order to establish the improvement in language learning. The second approach focused its quantitative model to determine the exact number of students who exceed learning expectations. Results. The meaningful learning approach applied in the present study showed that the level of English in higher education students were conducted with ideal results, since it allowed to determine how to maintain interest in developing communication strategies and improving writing and oral language skills. In the

\footnotetext{
${ }^{1}$ Escuela Superior Politécnica de Chimborazo, Facultad de Informática y Electrónica, Riobamba, Ecuador. lo.yumig@espoch.edu.ec, https://orcid.org/0000-0003-2282-2462

2 Escuela Superior Politécnica de Chimborazo, Facultad de Administración de Empresas, Riobamba, Ecuador. jose.andrade@espoch.edu.ec, https://orcid.org/0000-0003-3317-7219

3 Escuela Superior Politécnica de Chimborazo, Facultad de Administración de Empresas. Riobamba, Ecuador. natali.padilla@espoch.edu.ec,https://orcid.org/0000-0001-9355-7163
} 
collection of information sources, it was necessary to apply questionnaires, surveys and oral evaluations in the English language, since they allowed obtaining accurate results. Conclusion. It was concluded that the construction of unique material represented a great advance for the significant learning of the foreign language, cooperative work and the educational model based on experiences lived by university students allowed the implementation of a unique model when making their own comic strips.

Keywords: storytelling, communicative English, comic, strips, higher education.

\section{Resumen.}

Introducción. El aprendizaje significativo tiene un gran impacto en el proceso de enseñanza y aprendizaje del idioma inglés, el presente estudio fomenta la participación de estudiantes y docentes de lengua extranjera para la optimización en la producción oral y escrita de la lengua inglesa en la realización de material pedagógico genuino en a través de la creación de tiras Cómicas y la escritura inglesa. Objetivo. Demostrar la motivación de los estudiantes a nivel superior para el desarrollo lingüístico. Incentivar la creación de material ilustrativo para fomentar el dominio de lenguaje extranjero. Impulsar la producción oral a través de actividades como storytelling y communicative writing. Metodología. La investigación se condujo a través de dos métodos, el primero se basa en el método cualitativo para establecer la mejoría de aprendizaje del idioma. El segundo enfoque centra su modelo cuantitativo para determinar la cantidad exacta de estudiantes que superan las expectativas de aprendizaje. Resultados. El enfoque del aprendizaje significativo aplicado en el presente estudio demostró que el nivel de inglés en estudiantes de educación superior fue conducido con óptimos resultados, ya que permitió determinar cómo mantener el interés en desarrollar estrategias comunicativas y el mejoramiento de habilidades escrituras y de lenguaje oral. Al recabar las fuentes de información fue necesario la aplicación de cuestionarios, encuestas y evaluaciones orales en el idioma inglés ya que permitieron obtener los resultados certeros. Conclusión. Se concluyó que la construcción de material único represento un gran avance para el aprendizaje significativo del idioma extranjero, el trabajo cooperativo y el modelo de educativo basado en experiencias vividas por parte de estudiantes universitarios permitieron la implementación de un modelo único al realizar sus propias tiras cómicas.

Palabras clave: Cuentacuentos, inglés comunicativo, historietas, tiras, educación superior.

\section{Introducción.}

La aplicación de un verdadero aprendizaje significativo envuelve al entorno del aula más productivo, dinámico y enérgico; es decir el rol del estudiante es de carácter activo y la relación de sus conocimientos previos tienen que ser impulsados por la creatividad, empatía y dinámica como es conducida la cátedra dictada a nivel superior, en este ámbito educativo nos referimos al del aprendizaje del idioma inglés como lengua extranjera, para efectos de una acertada investigación fue necesaria mantener la colaboración de 
estudiantes de tercer semestre de la carrera de diseño gráfico pertenecientes a la Facultad de Informática y Electrónica de la Escuela Superior Politécnica de Chimborazo en el periodo académico especial. La motivación en la producción autentica de material didáctico como herramienta principal de aprendizaje aplicada a una segunda lengua motivó a estudiantes universitarios en investigar más allá de sus conocimientos previos, ya que la asistencia de docentes universitarios tanto de carácter inherente a la materia de inglés significo un importante apoyo para el desarrollo de los contenidos tales como gramática inglesa, vocabulario, y habilidades orales. Por otro lado, la asistencia de docentes propios de la carrera de diseño fue de un gran aporte para el desarrollo creativo digital del mismo.

Cabe mencionar como los desafíos en la educación superior impulsan la conexión que existen entre la adquisición de nuevos conocimientos a través de la aplicación de tecnologías digitales que envuelve la educación a superior en romper los paradigmas tradicionales donde la comunicación lingüística en el idioma ingles se refleja como la puerta a un nuevo mundo (Levicoy, 2013). En el mismo contexto, el aprendizaje significativo es una herramienta fundamental a través de distintas herramientas tecnológicas, a lo cual el autor enfatiza que es imperativo el aprender a convivir con los medios digitales e impulsar el ámbito educativo, el mejorar el desarrollo comunicativo representa el comprender sus medios y entrelazar sus contenidos.(Ralón, 2004)

En los momentos tan difíciles que atraviesa la educación superior, es entendible que el medio más accesible para un aprendizaje significativo es la utilización de las tecnologías de la información y comunicaciones conocidas muy comúnmente como TIC's, para lo cual su función principal se ve cristalizado como herramienta eficaz que conecta información digital y conocimientos que se explotan en el entorno del aula. El elemento clave para el manejo correcto de las TIC'S y la vinculación de una segunda lengua permita desarrollar nuevas estrategias didácticas. (Colás, 2002)

Por otro lado, en el sentido de perfeccionamiento de los contenidos del idioma extranjero conjuntamente con la motivación del aprendizaje del inglés, el desarrollo de inteligencias múltiples, Gardner enfatiza que un aprendizaje significativo se encuentra conectado a través de los sentidos, en este punto de estudio es conducido por la combinación de inteligencia viso espacial que su objeto principal es el análisis gráfico de comics strips, y un aprendizaje basado en inteligencia lingüística para la construcción de cada dialogo e historia del mismo. (Gardner, 2011).

Citando a Armstrong (2009), ratifica en su estudio que cada vez existen el incremento de aprendices quienes van descubriendo acorde al nivel de competencia y desarrollo de cada inteligencia esto sugiere que el desarrollo de habilidades innato en cada ser humano pero debe ser notablemente explotada acorde el desarrollo intelectual y motivacional del individuo, afianzando la teoría de un análisis por mayor de los conocimientos previos de cada participante de este estudio.

Cimermanová (2015) enfatiza que la manera de recibir los contenidos de estudios de una materia a nivel superior, ha cambiado el paradigma elocuentemente, puesto que la 
información es mucho más viable en estos días con el acceso de medios digitales, pero en la réplica para el campo didáctico demuestra una serie de problemas, esto no facilita al aprendizaje significativo y tan solo se enfoca en un aprendizaje directo y menos activo, es decir el que se obtiene de fuentes no seguras a través del internet sin mucho esfuerzo. Toh (2017), enfatiza en su estudio que el uso indicado de los comics como estrategia educativa en el proceso de enseñanza en el aula, permite explicar cómo los estudiantes se prepara para los retos que conlleva el siglo veintiuno, es así que el autor reúne su esfuerzo en demostrar a través de su estudio que la utilización de los ilustraciones gráficas prepara a los estudiantes para un mejor entendimiento.

Bajo el mismo ámbito de estudio, una de las estrategias más utilizadas para el desarrollo de competencias lingüísticas y motivar a la producción de materia autentico es definitivamente "Sotytellign" su visión de crear oportunidades de aprendizaje a través de la narración de historias a través del inglés como lengua extranjera impulsa al cambio de la clase tradicional contemporánea en una cosmovisión de estudio con proyección al futuro donde el estudiante es participe en todo momento.(Kukkonen, 2013)

Dicho con palabras de Mart (2013), el proceso más complejo en la producción de habilidades lingüísticas en el idioma inglés sin duda es la estructura gramatical, su grado de complejidad y el tiempo que conlleva a su desarrollo tiene a ser una de las habilidades difíciles de ser analizadas a su totalidad, es por ello que un aprendizaje efectivo obligatoriamente debe ser conducción a través de la experiencia propia y compartida con el aprendiz para que puedan tener la oportunidad de percibir dichas estructuras complejas de mejor manera.

Cada vez, el incremento de investigadores se refleja en una ata demanda, ellos suman sus esfuerzo para demostrar la importancia de la utilización de comics strips en el entorno educativo actual, a juicio de Engler (2008), menciona que existe un camino amplio donde los retos de enseñanza en idiomas extranjeros se ven abocados en el desarrollo a través de material académico propio donde los estudiantes forman parte y permiten aligerar la carga docente, estos programas académicos construyen un puente de comunión entre educadores a nivel superior de lengua extranjera con su modelo de aprendizaje donde sus receptores pueden ilustrar sus pensamientos a través de ilustraciones gráficas.

Al reforzar la adquisición y construcción de habilidades cognitivas en el idioma inglés es de gran beneficio el reforzar el incremento de vocabulario y gramática comunicativa, es por ello que son apreciados como pilar primordial en el desarrollo del idioma extranjero (Cabrera, 2018).

McVicker (2007), expresa en su investigación que a la estrategia didáctica denominada Comic Strip brinda la opción de ayuda al desarrollo de habilidades visuales combinado con el desarrollo de relatar cuentos, de la misma manera permite a docentes a universitarios como herramienta de gran apoyo, ya que motiva al desarrollo lectora y comprensiva en el idioma extranjero. Cabe remarcar que una de las habilidades más 
dinámicas para el aprendizaje de una segunda lengua es la lectura comprensiva, Sari Manik (2019) revela que el puntal con mayor incidencia es la lectura en comics, ya que el material desarrollado en este medio envuelve la predominio de comprensión lectora y el desarrollo de la inteligencia espacial, donde los docentes explotan a totalidad la creatividad del estudiante y comparten un aprendizaje atractivo para ambos. De igual manera, Rengur \& Sugirin (2019) manifiesta que la lectura en comics representa una de las más enérgicas de las habilidades en el en un segundo idioma, ya que motiva al lector extraer el significado de cada tira cómica, y obtener su comprensión única, a la par desarrollar actividades de lectura comprensiva.

Los Comic strips son muy notorias y de fácil acceso, es decir como herramientas didácticas más habituales para el aprendizaje se las puede encontrar en revistas y páginas web en la internet, que permiten la incorporación de noticias reales transformadas en ilustraciones gráficas, pero es necesario encontrar el modelo indicado para poder desarrollar de manera óptima el proceso de comunicación en el idioma deseado.(Özdemir, 2017)

\section{Metodología.}

La metodología investigativa aplicada mantienen dos enfoques transcendentales que nos permiten despejar nuestras inquietudes referentes a nuestro tema de estudio, al referirnos al enfoque cualitativo, Sisto (2008) menciona que este modelo metodológico antepone la relación existente entre los datos recabados de la investigación, que en este caso nuestro objetivo principal es determinar la calidad de aprendizaje de los estudiantes de diseño gráfico a través de la utilización de cómic strips inmersos en el idioma inglés. Por otro lado, concomitantemente el método cuantitativo nos permite cuantificar el determinado número de estudiantes que obtuvieron resultados asertivos en el proceso de enseñanza aprendizaje de esta segunda lengua. (García Cabrero \& Richard Martíez, 1996)

En el análisis de la investigación cuantitativa, fue necesario la participación de dos grupos de estudiantes de diseño gráfico, el primero grupo comprendían de 15 estudiantes elegidos de manera aleatoria, quienes conformaron el "Grupo de control", al cual se subdividieron en 5 equipos de trabajo, con miembros de 3 integrantes cada uno, quienes reunieron esfuerzos en el estudio del idioma ingles de la manera tradicional y rutinaria basados en reading y writing and speaking.

Un segundo equipo de trabajo, conformado por 15 estudiantes universitarios pertenecientes a la misma carrera, fueron escogidos de manera aleatoria, este equipo de trabajo se denominó "Grupo experimental", quienes focalizaron su trabajo en la implementación de Comics strips a través del idioma inglés fomentando la producción de Reading (strory tellign), writing (academic writing), speaking (communicative speech). (Ver figuras 1 y 2) 


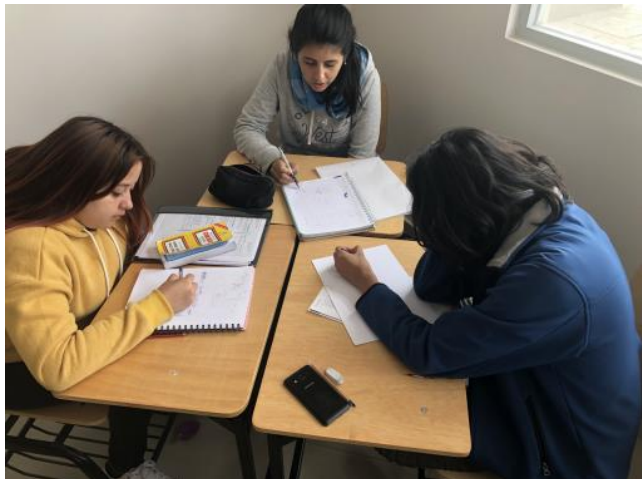

Figura 1. Grupo Control

Fuente: Escuela de Diseño Gráfico

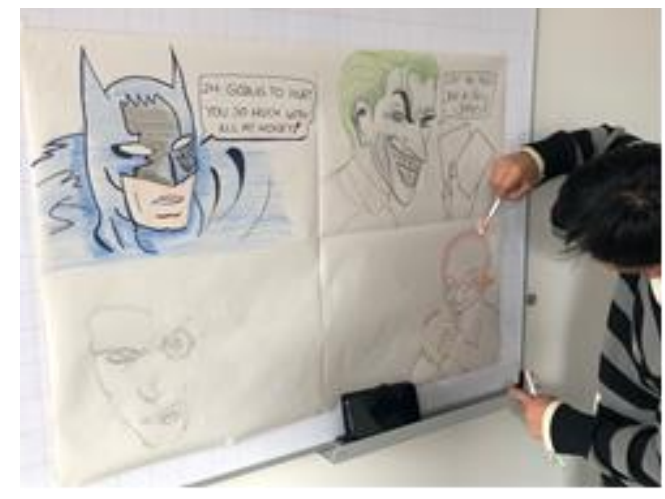

Figura 2. Grupo Experimental

Fuente: Escuela de Diseño Gráfico

En el siguiente cuadro de rubrica se puede evidenciar los parámetros de evaluación, que motivaron el estudio de la presente investigación, cabe indicar que todos los participantes quienes cooperaron de manera espontánea pertenecen a la Escuela Superior Politécnica de Chimborazo de la ciudad de Riobamba, provincia de Chimborazo.

Tabulación de datos en base a rubrica aplicada en ambos grupos de estudio, donde 1 representa menor valor y 5 es el valor mayor. (Ver tabla No. 1)

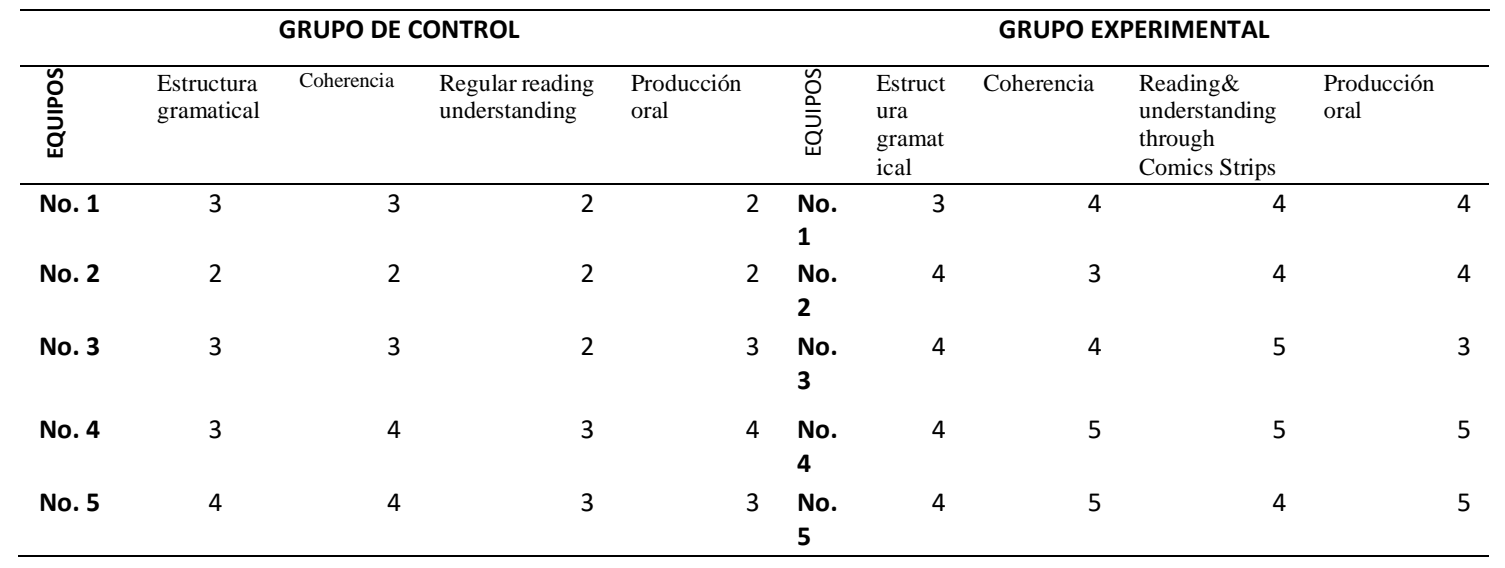

Tabla 1. Grupos de Control y Experimental

Fuente: Elaboración Propia

El proceso de evaluación aplicada a los participantes de esta investigación fue realizado en dos equipos, con la presencia de 2 docentes de lengua inglesa, en el proceso de monitoreo de la clase, bajo este contexto nos referimos al grupo de control quienes recibieron directamente la información y su réplica fue evidenciada acorde los resultados siguientes (Ver Figura No. 3). 


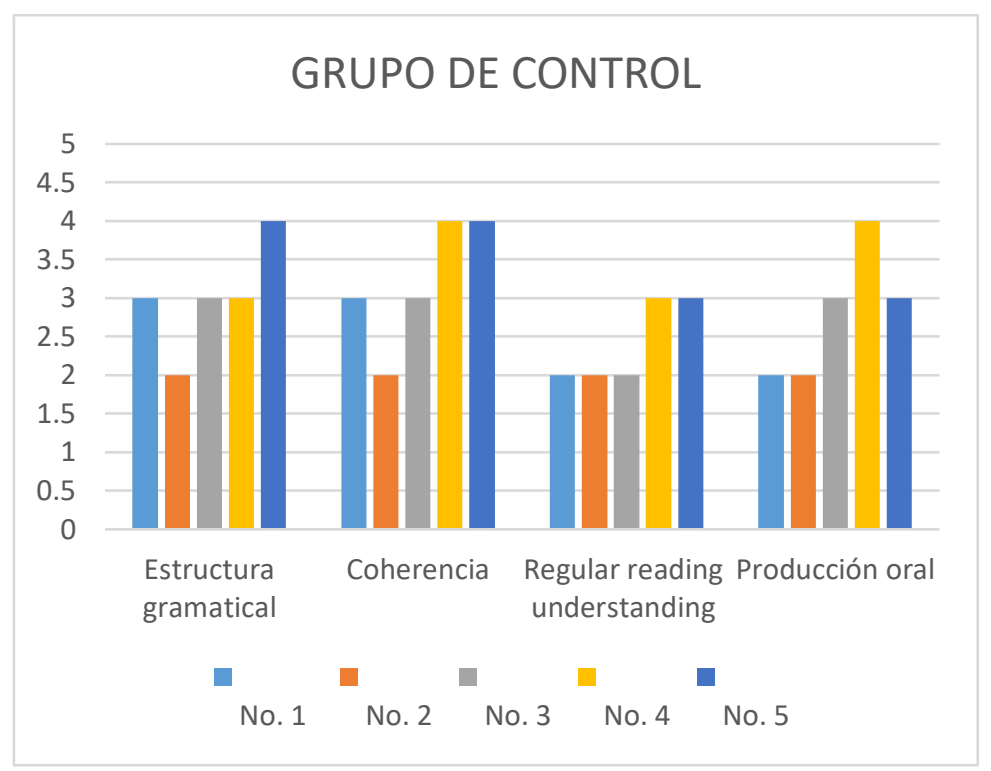

Figura 3. Grupo Control

Fuente: Elaboración Propia

El equipo No. 1 perteneciente al grupo de control su evaluación fue tres puntos sobre cinco en estructura gramatical, con respecto al ítem denominado coherencia su resultado fue de tres sobre cinco, en regular Reading\&understanding el desempeño fue de dos sobre cinco puntos, y la fluidez y producción oral el resultado obtenido fue de dos sobre cinco puntos. Cabe resaltar que estos resultados son bajos, debido a la falta de material didáctico y motivación extrínseca para el aprendizaje. Castillo (2009) enfatiza en su estudio que la motivación intrínseca es la clave de la apertura a nuevas oportunidades para todos los niveles de educación, en especial las herramientas didácticas aplicadas en el entorno del aula.

Con respecto al Equipo No.2 del grupo de control, se constató que en estructura gramatical el desempeño bajo el cual fue de dos sobres cinco puntos, en coherencia fue de dos sobre cinco puntos, regular reading\&understanding fue de dos sobre cinco puntos, y en producción oral en el lenguaje inglés se mantuvo con un desempeño no muy favorable de dos sobre cinco puntos.

El desempeño del Equipo No.3 del grupo de control pudo demostrar en estructura gramatical un desempeño aceptable de test sobre cinco puntos, la coherencia en su contenido fue de tres sobre cinco puntos, pero al producir regular reading\&understanding su resultado fue de dos puntos sobre cinco, y su producción oral fue de tres puntos sobre cinco.

En cambio, el rendimiento correspondiente al equipo No.4 el resultado de su desempeño fue de tres sobre cinco puntos, en coherencia su resultado es aceptable con cuatro sobre cinco puntos, en regular reading\&understanding es de tres puntos sobre cinco, sin embargo, su producción oral mejora con un equivalente de 4 puntos sobre cinco. 
Finalmente, el equipo No. 5 del grupo de control demostró en estructura gramatical un manejo apropiado del idioma inglés con un desempeño de cuatro sobre cinco puntos, su coherencia fue de cuatro puntos sobre cinco, en regular reading\&understanding su resultado fue de tres sobre cinco puntos y su producción oral fue de tres sobre cinco.

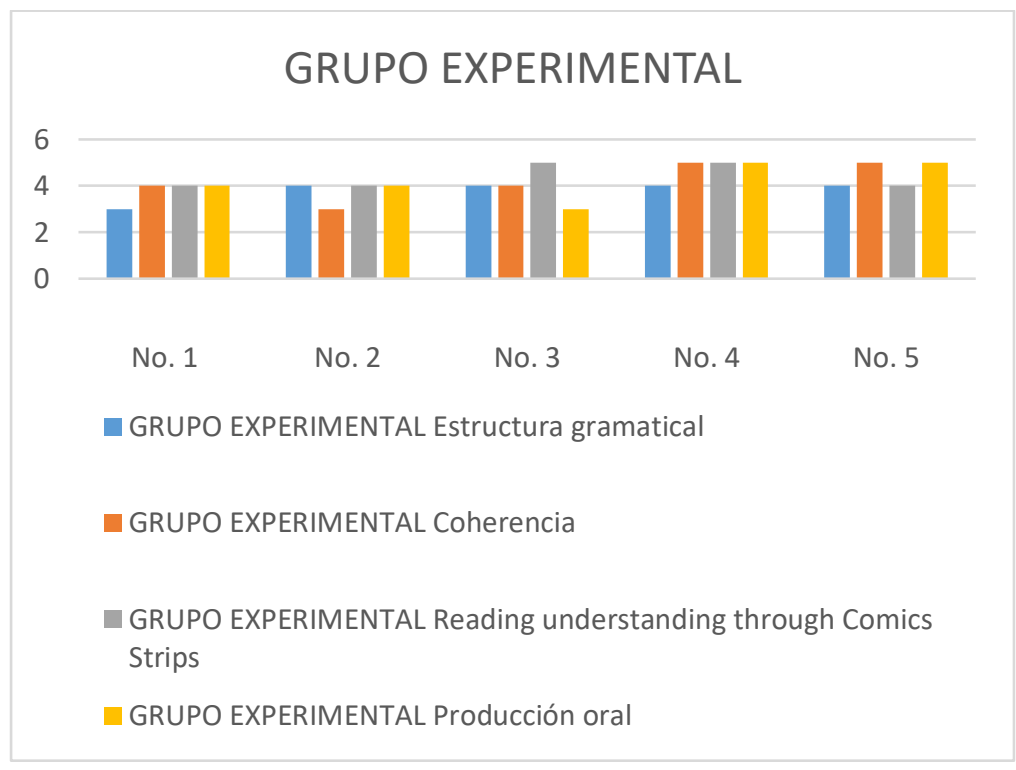

Figura 4. Grupo Experimental

Fuente: Elaboración Propia

Al entrar en detalle según los datos obtenidos del grupo Experimental, se pudo evidenciar que el equipo No. 1 su desempeño en estructura gramatical se mantiene con un indicador de tres puntos sobre cinco, en coherencia mejora su desempeño con cuatro sobre cinco puntos, en reading\&understanding through comics es de cuatro puntos sobre cinco y su producción oral mejora con un resultado de cuatro puntos sobre cinco. De la misma manera el equipo No. 2 mejora su desempeño con cuatro puntos sobre cinco en estructura gramatical, mientras que su coherencia fue de tres puntos sobre cinco, en el desempeño de Reading\&understanding through Comics Strips fue de cuatro sobre cinco, y su producción oral tubo una mejoría de 4 sobre cinco.

El equipo No. 3 en cuanto a su resultado en estructura gramatical fue de cuatro sobre cinco puntos, su coherencia fue de cuatro puntos sobre cinco, en el desarrollo de Reading\& understanding through Comics Strips fue una nota excelente de cinco sobre cinco puntos, y su producción se mantuvo en tres sobre cinco puntos.

El desempeño que se evidencio del equipo No. 4 en estructura gramatical fue de cuatro puntos sobre cinco, en su coherencia su resultado fue existo con cinco puntos sobre cinco, de la misma manera un resultado positivo en el desarrollo de Reading\& understanding 
through Comics Strips con cinco puntos sobre cinco, y su producción se notó la mejoría con cinco puntos sobre cinco.

Finalmente, el equipo No. 5 fue transcendental sus resultados en estructura gramatical con cuatro sobre cinco puntos, en coherencia fue excelente con cinco puntos sobre cinco, en el desarrollo de Reading\& understanding through Comics Strips fue cuatro sobre cinco puntos, y su producción se incrementó a la nota exacta de cinco puntos sobre cinco.

Para cristalizar de mejor manera el desempeño y progreso de los estudiantes quienes participaron en los grupos de control y experimental fueron encuestados con temas referentes al interés, ámbito motivacional y conocimiento del idioma, el cual se detalla a continuación

\section{Cuestionario aplicado a estudiantes de la escuela de diseño Gráfico, grupo de control y grupo experimental}

1.- En el proceso de enseñanza y aprendizaje con la ayuda de "comic strips" le resulto difícil?

2.-Le intereso la lectura y su deseo de aprender el idioma inglés a través de "comic strips"?

3.- Le resulta más fácil trabajar de la manera tradicional el idioma inglés?

4.- El desarrollo de actividades en el aula como trabajos grupales resultan ser más dinámicos al realizar actividades graficas?

5.- Se siente usted motivado al trabajar su propio material didáctico para el aprendizaje do idioma inglés?

6.- Cree usted que es inútil la aplicación de Comic strips como estrategia metodológica en el proceso de enseñanza y aprendizaje de una segunda lengua?

7.- ¿Cree usted que la aplicación de Comic strips en el aula son motivantes para la mejora en el aprendizaje de escritura, lectura y producción oral en la clase de inglés?

8.- Le resulta importante estudiar inglés en la carrera de diseño gráfico?

Después de dar por terminado la aplicación de evaluación a los dos grupos de trabajo en esta investigación se aplicó un cuestionario para determinar el progreso y adquisición de los contenidos en el idioma inglés a través de comic strips. El total de encuestados fueron treinta estudiantes, el detalle de cada pregunta se adjunta a continuación:

¿En el proceso de enseñanza y aprendizaje con la ayuda de comic strips al estudiante le resulto difícil? La respuesta fue de si 6,7\% y un 93,3\%, en lo que corresponde al interés en la lectura y el deseo de aprender el idioma inglés a través de "comic strips" respondieron con $96,7 \%$ si, y el 3,3\% respondieron no, referente a la pregunta 3 acerca de si al estudiante le resulta más fácil trabajar de la manera tradicional el idioma inglés? $86,7 \%$ respondieron que si, $13,3 \%$ respondieron que no. ¿Con respecto a la pregunta 4 que menciona "El desarrollo de actividades en el aula como trabajos grupales resultan ser 
más dinámicos al realizar actividades graficas?” 86,7\% respondieron que Sí, y un 3,33\% indicaron que no aplica. ¿En la pregunta 5 "Se siente usted motivado al trabajar su propio material didáctico para el aprendizaje do idioma inglés?" respondieron 86,7 que Sí, un $13,3 \%$ respondieron que No, en lo que corresponde a la pregunta 6 que menciona "Cree usted que es inútil la aplicación de Comic strips como estrategia metodológica en el proceso de enseñanza y aprendizaje de una segunda lengua?" un 10\% indicaron que sí, $83,3 \%$ dijeron que no, y 6,67\% respondieron que no aplica. En la pregunta 7 “ ¿Cree usted que la aplicación de Comic strips en el aula son motivantes para la mejora en el aprendizaje de escritura, lectura y producción oral en la clase de inglés?" 83,3\% indicaron que Sí, un $10 \%$ respondieron que No, y 6,67\% no aplica. Finalmente, ¿en la pregunta 8 menciona que si al estudiante le resulta importante estudiar inglés en la carrera de diseño gráfico? Un 90\% respondieron que Sí, un 6,7\% respondieron que No, y un 3,33 \% no aplica.

\section{Resultados.}

Al realizar un análisis profundo de los resultados obtenidos en la investigación, se determinó que la aplicación de material didáctico en el desarrollo de las actividades en el aula es de gran importancia, más de un 90 por ciento de los estudiantes coincidieron que la implementación de comic strips representan un avance para conducir las habilidades lingüísticas del idioma ingles y representó un impacto motivante en los estudiantes universitarios quienes participaron en el proceso y progreso de esta investigación.

En el desarrollo de actividades inherentes al estudio del idioma inglés se pudo evidenciar que el proceso de aprendizaje en nivel superior tiene un mejor desempeño cuando el estudiante es artífice de su aprendizaje y el docente motiva un aprendizaje más dinámico, la pedagogía y metodología claramente es responsabilidad del docente pero el desarrollar un material propio con todos los elementos dinámicos que mejoren la calidad de la docencia es la gran compromiso de ambos ya que dejamos a un lado el paradigma de un aprendizaje tradicional que limita el proceso de enseñanza.

Finalmente se determinó que el grupo de control demostró un rendimiento regular en sus actividades, la metodología y rutina de aprendizaje no permitió el desarrollo emocional e intelectual de sus participantes, poniendo en evidencia que el aprendizaje directo no es una buena estrategia en el aula.

\section{Conclusiones.}

No hay duda que el ser humano aprende de manera eficaz a través de un modelo significativo, experiencial y activo, donde la motivación nace desde su interior y en muchas ocasiones es provocado por factores externos como es en el entorno del aula, es decir los intereses de aprendizaje se adaptan acorde a las necesidades en su entorno, es así que la implementación de material didáctico a través de la elaboración de comics nos ha permitido concluir los siguientes puntos: 
- El proceso de aprendizaje del idioma inglés como segunda lengua a nivel superior se basa en la construcción de conocimientos a través experiencias propias del alumno, donde el modelo de aprendizaje significado permite desarrollar las habilidades del idioma ingles de mejor manera, en Speaking fluye sin contratiempos, en Reading tiene una mejor adaptación a situaciones reales, y en Academic writing permite al estudiante adaptar sus necesidades acordes a su carrera.

- EL desarrollo del comic strips incentiva la imaginación y la creatividad para acoplar a los temas analizados, es decir permite al alumno explorar más sus conocimientos previos hacia los nuevos.

- El manejo responsable de las tecnologías de la información en el ámbito educativo permite a los docentes y estudiantes universitarios expandir más su creación inédita de material didáctico en el aula, de esta manera se optimiza el tiempo pedagógico aplicado en el aula.

- Finalmente, se concluyó que el material didáctico en el aula no tiene limitación alguna sin embargo es necesario tener un control de su contenido y calidad al momento de aplicarlo, su valioso aporte permito que el aprendizaje se vea reflejado en los participantes de esta investigación permitiendo la apertura a nuevas estrategias metodológicas.

\section{Referencias bibliográficas.}

Armstrong, T. (2009). Multiple intelligencies in the classroom (Third edit). Alexandria.

Cabrera, P., Castillo, L., González, P., Quiñónez, A., \& Ochoa, C. (2018). The impact of using Pixton for teaching grammar and vocabulary in the EFL Ecuadorian context. Teaching English with Technology, 18(1), 53-76.

Castillo, A. C. (2009). El Papel De La Motivación Intrínseca, Los Estilos De Aprendizaje Y Estrategias Metacognitivas En La Búsqueda Efectiva De Información Online. Píxel-Bit. Revista de Medios y Educación, O(35), 73-85.

Cimermanová, I. (2015). Using Comics with Novice EFL Readers to Develop Reading Literacy. Procedia - Social and Behavioral Sciences, 174, 2452-2459. https://doi.org/10.1016/j.sbspro.2015.01.916

Colás, P. (2002). La investigación educativa en la (nueva) cultura científica de la sociedad del conocimiento. XXI Revista de Educación, 4(2002), 77-93. http://uhu.es/publicaciones/ojs/index.php/xxi/article/viewArticle/623

García Cabrero, J., \& Richard Martíez, M. (1996). El debate investigación cualitativa frente a investigación cuantitativa. Enfermería Clínica, 6(5), 212-217. http://xa.yimg.com/kq/groups/21897551/2125185069/name/AD_Cabrero_GarcíaMartinez_Unidad_2.pdf

Engler, S., Hoskins, C., \& Payne, S. (2008). Computer-produced comics as a means of summarizing academic readings in EAP programs. International Journal of Pedagogies and Learning, 4(4), 19-33. 
Gardner, H. (2011). Frames of Mind The theory of Multiple Intelligences (Second Edi). Basic Books.

Kukkonen, K. (2013). Contemporary comics storytelling. In Contemporary Comics Storytelling. https://doi.org/10.5860/choice.51-3667

Levicoy, D. D. (2013). TIC en Educación Superior: Ventajas y desventajas. Dialnet, $c$, 44-50. https://dialnet.unirioja.es/servlet/articulo?codigo $=5072156 \% 0 \mathrm{~A}$

Mart, C. (2013). Teaching grammar in context: Why and How? Theory and Practice in Language Studies, 3(1), 124.

McVicker, C. J. (2007). Comic Strips as a Text Structure for Learning to Read. The Reading Teacher, 61(1), 85-88. https://doi.org/10.1598/rt.61.1.9

Özdemir, E. (2017). Humor in elementary science: development and evaluation of comic strips about sound. International Electronic Journal of Elementary Education, 9(4), 837-850.

Ralón, L., Vásquez, L., \& Vieta, M. (2004). (De)formación en línea: acerca de las desventajas de la educación virtual. Comunicar: Revista Científica Iberoamericana de Comunicación y Educación, 22, 171-176. https://doi.org/10.3916/25599

Rengur, Z. A., \& Sugirin. (2019). The Effectiveness Of Using Comic Strips To Increase Students' Reading Comprehension For The Eight Grade Of SMPN 1 Pundong. 330(Iceri 2018), 239-243. https://doi.org/10.2991/icossce-icsmc-18.2019.24

Sari Manik, R. M. (2019). The Effectiveness of Comic Strip As Media to Improve the Students' Reading Comprehension on Narrative Text of MTs Ar-Rahman Langkat. KnE Social Sciences, 2019, 933-947. https://doi.org/10.18502/kss.v3i19.4918

Sisto, V. (2008). La investigación como una aventura de producción dialógica: Psicoperspectivas, 7, 114-136.

Toh, T. L., Cheng, L. P., Ho, S. Y., Jiang, H., \& Lim, K. M. (2017). Use of comics to enhance students' learning for the development of the twenty-first century competencies in the mathematics classroom. Asia Pacific Journal of Education, 37(4), 437-452. https://doi.org/10.1080/02188791.2017.1339344

\section{LC Ciencia}




\section{PARA CITAR EL ARTÍCULO INDEXADO.}

Yumi Guacho, L. M., Andrade Mendoza, J. L., \& Padilla Padilla, Y. N. (2021). Aprendizaje del inglés a través de tiras cómicas en educación superior ecuatoriana. ConcienciaDigital, $4(1.2)$,

286-298. https://doi.org/10.33262/concienciadigital.v4i1.2.1594

\section{LCiencia}

El artículo que se publica es de exclusiva responsabilidad de los autores y no necesariamente reflejan el pensamiento de la Revista Conciencia Digital.

El artículo queda en propiedad de la revista y, por tanto, su publicación parcial y/o total en otro medio tiene que ser autorizado por el director de la Revista Conciencia Digital.



